\title{
The Kinetics of Segregation and Its Application to the Sulfur on the Fe(100) Surface*
}

\author{
By Kazuhiro Yoshihara** and Kazuyoshi Nii**
}

\begin{abstract}
An iron single crystal with the bulk sulfur concentration adjusted between 23 and 66 mass ppm was heated at $1023 \mathrm{~K}$ in vacuum, and the segregation rate of sulfur on the (100) surface was measured with good reproducibility by using AES. The result was compared with a theoretical equation.

Because the equilibrium concentration of sulfur on the surface was constant, unaffected by the bulk sulfur concentration and annealing temperature, the number of the surface sites on which sulfur could segregate was assumed to be constant. Assuming further that the segregation rate is proportional to the number of vacant sites on the surface and the bulk concentration of the segregant beneath the surface, a theoretical equation of the segregation rate has been derived. This equation shows that at an early stage of segregation the concentration on the surface increases in proportion to the annealing time, and that the segregation rate is proportional to the bulk concentration and independent of the diffusion coefficient of the segregant.

From the experiments, the concentration of sulfur on the surface was observed to increase in proportion to the annealing time and then reached a constant value. The segregation rate became greater as the bulk sulfur concentration increased. This tendency coincides well with that of the theoretical equation.

A concentration profile of the segregant near the surface influenced the segregation rate at the first stage. Therefore, in order to observe the segregation behavior with good reproducibility, it was necessary prior to measurements to sufficiently anneal the sample at a measuring temperature and sputter the surface by argon ions until the concentration on the surface became equal to the bulk concentration.
\end{abstract}

(Received May 23, 1983)

Keyzords: iron, kinetics, segregation, sulfur, surface, vacuum

\section{Introduction}

In the course of heating of metals, impurities often segregate to interfaces and change the properties of metals. It is important to investigate the kinetics of segregation, in order to understand the mechanism of segregation.

McLean $^{(1)}$ supposed the relation between the surface concentration of segregant, $C_{\mathrm{s}}$, and the bulk concentration just beneath the surface, $C_{x=0}$, as follows;

$$
C_{\mathrm{s}} / C_{x=0}=\alpha=C_{\mathrm{so}} / C_{\mathrm{o}},
$$

where $C_{\mathrm{so}}$ is the equilibrium surface concentration attained after infinite time and $C_{0}$ is the bulk concentration far from the surface. The following initial condition was also sup-

* This paper was originally published in Japanese in J. Japan Inst. Metals, 43 (1979), 414.

** National Research Institute for Metals, Meguroku, Tokyo 153, Japan. posed by McLean;

$$
t=0, C=C_{0}, x>0
$$

and

$$
C=0, x=0,
$$

where $C$ is the concentration of segregant. He obtained a solution in the form of eq. (4) by solving Fick's equation (eq. (3)) on the assumption of eqs. (1) and (2). He neglected the loss of segregant from the surface by evaporation.

$$
\partial C / \partial t=D\left(\partial^{2} C / \partial x^{2}\right)
$$

and

$$
C_{\mathrm{s}}=C_{\text {so }}\left\{1-\exp \left(D t / \alpha^{2} d^{2}\right) \operatorname{erfc}(\sqrt{D t} / \alpha d)\right\},
$$

where $D$ is the volume diffusion coefficient of segregant and $d$ the thickness of the surface. Reinmuth $^{(2)}$ assumed the following adsorption isotherm in place of eq. (1);

$$
C_{\mathrm{s}} / C_{\mathrm{so}}=C_{x=0} /\left(C_{x=0}+a\right),
$$

where $a$ is an isotherm constant. On the as- 
sumption of eq. (5), the solution of Fick's equation was obtained in the following series form;

$$
C_{\mathrm{s}} / C_{\mathrm{so}}=\sum_{j=0}^{\infty} a_{j}\left(4 \pi a^{2} D t / C_{\mathrm{so}}^{2}\right)^{j / 2},
$$

where $a_{0}=0$ and $a_{1}=C_{\mathrm{o}} / a \pi$, and the following coefficients were given by the recurrent formula;

$j a_{j} B(j / 2,1 / 2)=-a_{j-1}+\sum_{i=2}^{j-1} i a_{i} a_{j-1} B(j / 2,1 / 2)$,

where $B(j / 2,1 / 2)$ is the Beta function.

From these kinetic equations, it is deduced that the equilibrium surface concentration depends on the bulk concentration. The equilibrium concentration of sulfur on the (100) surface of Fe, however, was independent of the bulk concentration ${ }^{(3)}$. Therefore, these kinetic equations cannot be applied to the present case. It is also hardly believable that $C_{\mathrm{s}}$ is in equilibrium with $C_{x=0}$ at any time.

Since the equilibrium concentration of sulfur on the (100) surface of $\mathrm{Fe}$ is constant and independent of annealing temperature and the bulk concentration ${ }^{(3)}$, the number of surface sites on which sulfur can segregate is assumed to be constant. Assuming further that the segregation layer is monolayer and the boundary condition is determined by the transfer from the bulk to the segregation layer, a theoretical equation of the segregation rate is derived from Fick's second law. This equation is compared with the observed segregation behavior of sulfur on the (100) surface of Fe. The reproducibility of the segregation behavior is also discussed.

\section{Theoretical Equation}

A depth profile of segregant concentration is assumed as shown in Fig. 1. When segregant diffuses in the bulk with volume diffusion coefficient, $D$, the change in the concentration of segregant is deduced from Fick's second law. On the assumption of eq. (2), $C_{x=0}$, the solution of Fick's second law at $x=0$, is shown in the form of eq. (8) from Duhamel's theorem $^{(4)}$.

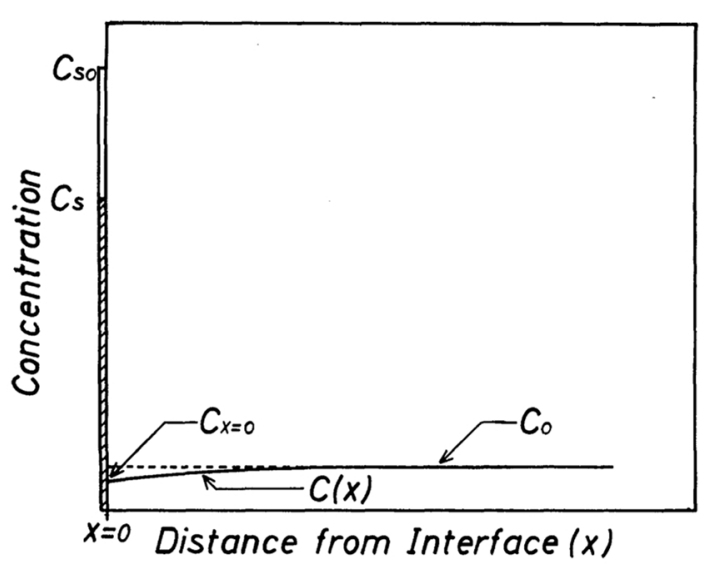

Fig. 1 The approximate concentration profile for deriving the theoretical equation.

$C_{x=0}=C_{0}-(D / \pi)^{1 / 2} \int_{0}^{t}(\partial C / \partial x)_{x=0}(t-\tau)^{-1 / 2} d \tau$

where $\tau$ is an integration variable.

In the present paper, the number of surface sites on which sulfur can segregate is assumed to be constant. The boundary condition is also determined by the following assumption; the segregation rate is proportional to the number of vacant sites on the surface and the bulk concentration just beneath the surface, as shown in eq. (9),

$$
d C_{\mathrm{s}} / d t=k\left(C_{\mathrm{so}}-C_{\mathrm{s}}\right) C_{x=0},
$$

where $C_{\mathrm{s}}$ is the surface concentration of segregant and $C_{\text {so }}$ is the equilibrium surface concentration and $k$ is the rate constant. It is also assumed that the loss of segregant by the evaporation is negligible so that the material flux at the boundary is equal to the rate of segregation:

$$
d C_{\mathrm{s}} / d t=D(\partial C / \partial x)_{x=0} .
$$

Equations (8), (9) and (10) can be combined to give eq. (11) for $C_{\mathrm{s}}$.

$$
\begin{aligned}
& \left(d C_{\mathrm{s}} / d t\right) / k\left(C_{\mathrm{so}}-C_{\mathrm{s}}\right) \\
& \quad=C_{\mathrm{o}}-(\pi D)^{-1 / 2} \int_{0}^{t}\left(d C_{\mathrm{s}} / d t\right)(t-\tau)^{-1 / 2} d \tau .
\end{aligned}
$$

In this paper, the solution of eq. (11) is obtained by the same method as that of Reinmuth $^{(2)}$. It is convenient to define the following parameters; 


$$
\mu=C_{\mathrm{s}} / C_{\mathrm{so}}, \psi=k C_{\mathrm{o}} \text { and } \theta=4 \pi D t / k^{2} C_{\mathrm{so}}^{2} \equiv \eta t
$$

Equation (11) can then be written in the form

$$
(d \mu / d t) /(1-\mu)=\psi-2 \int_{0}^{\theta}(d \mu / d \rho)(\theta-\rho)^{-1 / 2} d \rho \text {. }
$$

Equation (12) can be solved by assuming the solution to be of the form

$$
\mu=\sum_{0}^{\infty} a_{j} \theta^{j / 2} \equiv \sum_{0}
$$

From this it follows that

$$
d \mu / d \theta=(2 \theta)^{-1} \sum_{1}^{\infty} j a_{j} \theta^{j / 2} .
$$

Substituting eqs. (13) and (14) for eq. (12) and defining a new integration variable, $v=$ $\rho / \theta$, the following equation is obtained;

$$
\begin{aligned}
& \eta(2 \theta)^{-1} \sum_{1}^{\infty} j a_{j} \theta^{j / 2} /\left(1-\sum_{0}\right) \\
& \quad=\psi-\theta^{-1 / 2} \sum_{1}^{\infty} j a_{j} \theta^{j / 2} \int_{0}^{1} v^{j / 2-1}(1-v)^{-1 / 2} d v
\end{aligned}
$$

and

$$
\int_{0}^{1} v^{j / 2-1}(1-v)^{-1 / 2} d v=B(j / 2,1 / 2)
$$

where $B(j / 2,1 / 2)$ is the Beta function. Therefore, eq. (15) can be written in the form

$$
\begin{aligned}
& \eta(2 \theta)^{-1} \sum_{1}^{\infty} j a_{j} \theta^{j / 2}+\sum_{0} \\
& =\left(1-\sum_{0}\right)(\psi-\theta)^{-1 / 2} \cdot \sum_{1}^{\infty} j a_{j} \theta^{j / 2} B(j / 2,1 / 2) .
\end{aligned}
$$

The coefficients, $a_{j}$, can be readily evaluated by equating terms with equal powers of $\theta$ in eq. (17). Because $\mu$ is zero at $t=0$, the first coefficient is

$$
a_{0}=0 \text {. }
$$

Therefore, the second and the third coefficients are

$$
a_{1}=0 \text {, }
$$

and

$$
a_{2}=\psi / \eta,
$$

and the following coefficients are given by the recurrent formula,

$$
\begin{aligned}
(j+2) \eta a_{j+2} / 2= & -(j+1) a_{j+1} B\{(j+1) / 2,1 / 2\} \\
& +\sum_{i=1}^{j-1}(j-i+1) a_{j-i+1} a_{i} \\
& \times B\{(j-i+1) / 2,1 / 2\}-\psi a_{j} .
\end{aligned}
$$

Therefore, $C_{\mathrm{s}}$ is given by the following equation;

$$
\begin{aligned}
C_{\mathrm{s}} / C_{\mathrm{so}}= & k C_{\mathrm{o}} t-\left(4 k^{2} C_{\mathrm{o}} C_{\mathrm{so}} / 3 \sqrt{\pi D}\right) t^{3 / 2} \\
& +\left\{k^{2} C_{\mathrm{o}}\left(k C_{\mathrm{so}}^{2}-D C_{\mathrm{o}}\right) / 2 D\right\} t^{2}-\cdots
\end{aligned}
$$

Equation (22) shows that $C_{\mathrm{s}}$ increases in proportion to the annealing time at $t \simeq 0$, and the segregation rate is in proportion to the initial bulk concentration and independent of the volume diffusion coefficient.

Substituting the values of $k, C_{0}, C_{\text {so }}$ and $D$ for eq. (22), the relation between $C_{\mathrm{s}}$ and the annealing time is obtained.

\section{Experimental}

A specimen used was an iron single crystal (100) prepared by the strain-anneal method, and its sulfur concentration was adjusted between 23 and 66 mass ppm. The purity of the specimen and the adjusting method of sulfur concentration were stated elsewhere ${ }^{(3)}$.

The specimen was polished with emery paper and diamond paste. After being washed in acetone, it was attached to a holder ${ }^{(3)}$, and then it was installed in an AES apparatus (PHI 10-155). The specimen was heated by electron bombardment from the back side. The temperature was measured with a thermocouple which was spot-welded on the surface. Drift of the temperature was within $\pm 3 \mathrm{~K}$ at $1100 \mathrm{~K}$.

After the temperature of the specimen was raised, the surface was sputtered with argon ions to be cleaned. Then the segregation behavior of sulfur on the surface was continuously observed with AES. The surface activity of sulfur is larger than other segregants such as oxygen and phosphorus, so that the segregation behavior of sulfur is not affected by other segregants ${ }^{(3)}$. Therefore, discussion was restricted on the segregation behavior of sulfur in the present paper. 


\section{Results and Discussion}

\section{Check of reproducibility}

After the specimen containing 45 mass ppm sulfur was annealed at $1023 \mathrm{~K}$ for $54 \mathrm{ks}$, the surface was sputtered by argon ions. The effect of sputtering time on the segregation behavior is shown in Fig. 2. The surface concentration of sulfur reached a constant value, $28 \mathrm{at} \%$, and was independent of sputtering time. When the acceleration voltage was $2 \mathrm{kV}$ and the current density of argon ions was $0.03 \mathrm{~A} / \mathrm{m}^{2}$, the segregation behavior after sputtering for $240 \mathrm{~s}$ was quite similar to that after sputtering for $480 \mathrm{~s}$. The surface concentration of sulfur increased in proportion to the annealing time. When the acceleration voltage was $0.5 \mathrm{kV}$ and the current density of argon ions was $0.02 \mathrm{~A} / \mathrm{m}^{2}$, it took $180 \mathrm{~s}$ to clean the surface by sputtering. After this sputtering, the rate of sulfur segregation was large at the initial stage, but gradually became small and similar to that after sputtering with the acceleration voltage of $2 \mathrm{kV}$ and the current density of $0.03 \mathrm{~A} / \mathrm{m}^{2}$.

The reason why the segregation rate was influenced by the sputtering condition was assumed as follows: The initial surface concentration of sulfur was equal to the bulk concentration, when the surface was cleaned with sputtering with the acceleration voltage of $2 \mathrm{kV}$ and the current density of $0.03 \mathrm{~A} / \mathrm{m}^{2}$. However, after sputtering with the acceleration voltage of $0.5 \mathrm{kV}$ and the current density of

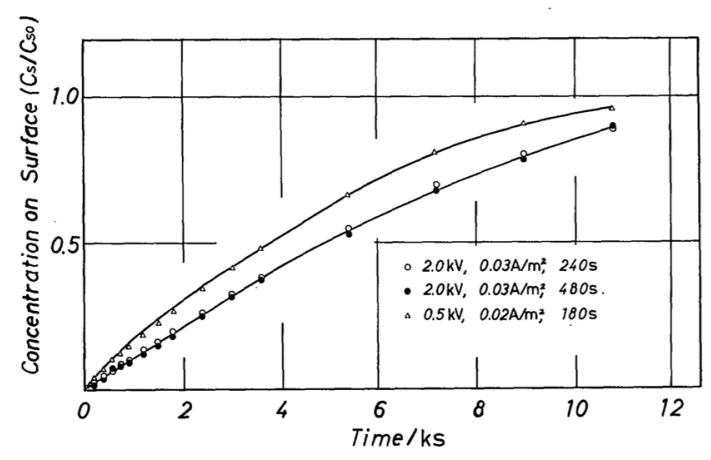

Fig. 2 The segregation behavior of the annealed sample under various sputtering conditions.

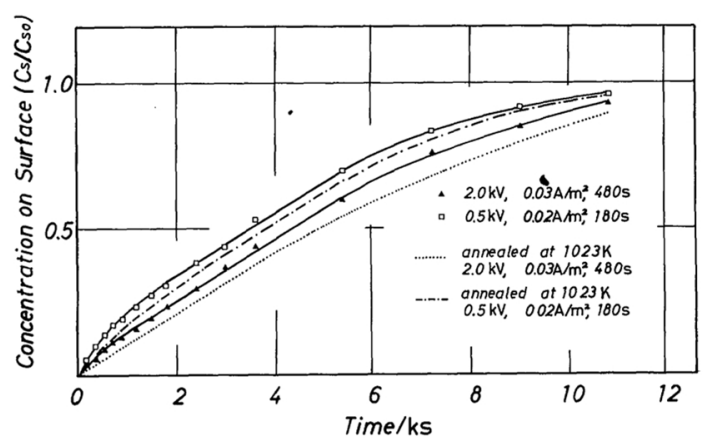

Fig. 3 The segregation behavior of the quenched sample under various sputtering conditions.

$0.02 \mathrm{~A} / \mathrm{m}^{2}$, the initial surface concentration of sulfur might not be reduced to the bulk concentration. The surface concentration below about 1 at $\%$ cannot be detected by AES. Therefore, these increased sulfur might influence the segregation rate at the initial stage.

The specimen was annealed at $1073 \mathrm{~K}$ for $10.8 \mathrm{ks}$ and then quenched to $1023 \mathrm{~K}$. After the quenching, the surface was cleaned with sputtering, then the segregation behavior was observed. The results are shown in Fig. 3. The segregation rate of the quenched specimen was compared with that of the specimen annealed at $1023 \mathrm{~K}$ for $54 \mathrm{ks}$. The initial segregation rate of the quenched specimen was slightly larger than that of the annealed specimen when sputtered for $480 \mathrm{~s}$ with argon ions with the acceleration voltage of $2 \mathrm{kV}$ and the current density of $0.03 \mathrm{~A} / \mathrm{m}^{2}$. The initial segregation rate of the quenched specimen, however, was much larger than that of the annealed specimen when sputtered for $180 \mathrm{~s}$ with argon ions with the acceleration voltage of $0.5 \mathrm{kV}$ and the current density of $0.02 \mathrm{~A} / \mathrm{m}^{2}$. The annealing or sputtering conditions had no effect on the segregation behavior at the final stage.

In this paper, the depth profile of segregant concentration has been assumed to be independent of the annealing temperature, for ease in solving Fick's equation. Actually, however, the depth profile at $1023 \mathrm{~K}$ is possibly sharper than that at $1073 \mathrm{~K}$, as shown in Fig. 4. Therefore, the different annealing and the different sputtering time give the different initial surface concentration. These differences are shown schematically as point $\mathrm{A}$ and point 


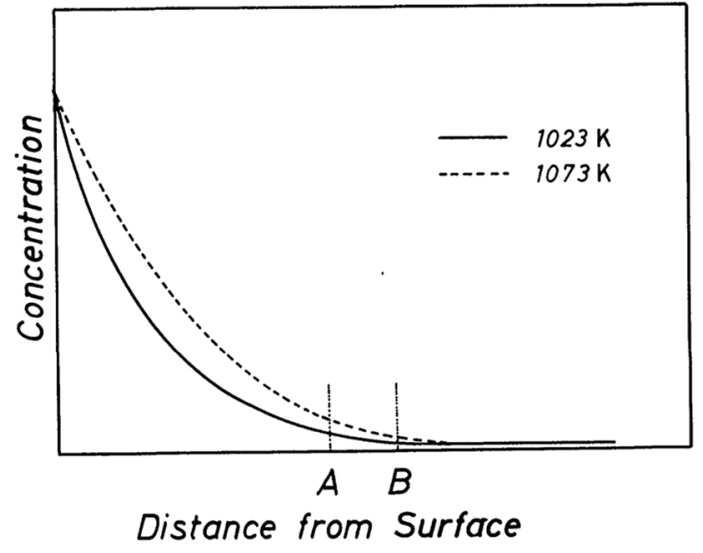

Fig. 4 The approximate representation of the concentration profile at $1023 \mathrm{~K}$ and $1073 \mathrm{~K}$.

$B$ in Fig. 4. The initial surface concentration of the specimen annealed at $1073 \mathrm{~K}$ is larger than that of the specimen annealed at $1023 \mathrm{~K}$, and the difference between these initial surface concentrations is larger, as the amount removed by sputtering is smaller. Therefore, the larger initial surface concentration of the specimen quenched from $1073 \mathrm{~K}$ to $1023 \mathrm{~K}$ provides a larger segregation rate than that of the specimen well annealed at $1023 \mathrm{~K}$. The effect of this difference between the initial surface concentrations becomes smaller, as the heating time is prolonged, and the segregation rate of the quenched specimen becomes similar to that of the annealed specimen after about $500 \mathrm{~s}$.

\section{Comparison with the thoretical equation}

Equation (22) is approximately expressed as eq. (23) at $t \simeq 0$.

$$
C_{\mathrm{s}} / C_{\mathrm{so}} \simeq k C_{\mathrm{o}} t \equiv K t .
$$

According to eq. (23), the concentration of segregant increases in proportion to the annealing time at $t \simeq 0$. The segregation rate, $K$, increases in proportion to the bulk concentration, $C_{0}$, and is independent of the volume diffusion coefficient, $D$, at $t \simeq 0$.

After the specimen had been annealed at $1023 \mathrm{~K}$ for $54 \mathrm{ks}$, the surface of the specimen was sputtered by argon ions with the acceleration voltage of $2 \mathrm{kV}$ and the current density of $0.03 \mathrm{~A} / \mathrm{m}^{2}$. In this case, the concentration of segregant increased in proportion to the annealing time. This result coincided with eq. (23). The segregation rate, $K$, of the specimen, the bulk concentration of which was 45 mass ppm, was $1.05 \times 10^{-4} \mathrm{~s}^{-1}$ at $1023 \mathrm{~K}$. Then $k$ was $1.5 \times 10^{-29} \mathrm{~m}^{3}$ atom ${ }^{-1} \mathrm{~s}^{-1}$. The value of $k$ means how frequently atoms jump onto the surface from the bulk, but it is difficult to calculate this value theoretically. Therefore, the experimental value of $k$ is used to calculate eq. (22).

It is also necessary to obtain the value of $C_{\text {so }}$ to calculate eq. (22). The (100) surface structure of $\mathrm{Fe}$ satulated with sulfur is that two iron atoms combined with one sulfur atom ${ }^{(3)}$. Therefore, sulfur occupies $1 / 2$ of surface sites. The volume diffusion coefficient of sulfur in iron was measured by Ainslie ${ }^{(5)}$. The values used for the calculation are as follows;

$$
\begin{aligned}
& k=1.5 \times 10^{-29} \mathrm{~m}^{3} \text { atom }{ }^{-1} \mathrm{~s}^{-1}, \\
& C_{\mathrm{o}}=45 \text { mass ppm }=6.6 \times 10^{24} \text { atom m} \mathrm{m}^{-3}, \\
& C_{\mathrm{so}}=1 / 2 \text { monolayer }=6.1 \times 10^{18} \text { atom m } \mathrm{m}^{-2},
\end{aligned}
$$
and

$$
D=6.0 \times 10^{-15} \mathrm{~m}^{2} \mathrm{~s}^{-1} \text {. }
$$

The result of calculation is shown in Fig. 5. The difference between theoretical values and experimental ones becomes greater, as the annealing time is prolonged. One of the assumptions for deriving the theoretical equation is that the segregation layer is a monolayer. Actually, however, the depth profile of segregant concentration will be expressed by an exponential function. Therefore, the thickness of segregation layer increases, as the annealing time is prolonged, as shown in Fig. 6. Then,

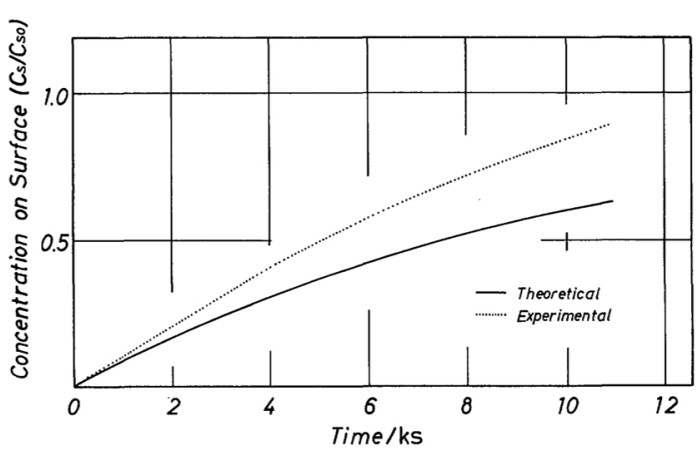

Fig. 5 The time dependence of the concentration of sulfur on the surface (theoretical and experimental). 


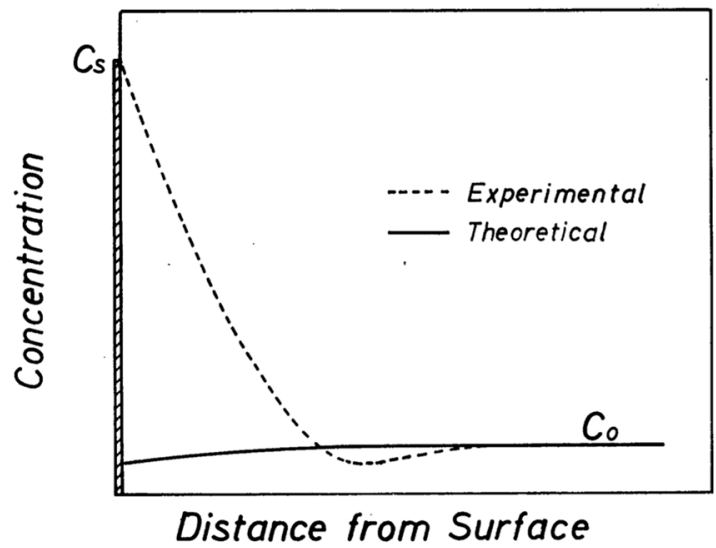

Fig. 6 The approximate representation of the concentration profile (theoretical and experimental).

the difference between theoretical values and experimental ones will be greater.

The bulk concentration of segregant at the interface, $C_{x=0}$, is calculated. Equations (8) and (11) can be combined to give eq. (24) for $C_{x=0}$,

$$
C_{x=0}=C_{\mathrm{o}}-(\pi D)^{-1 / 2} \int_{0}^{t}\left(d C_{\mathrm{s}} / d t\right)(t-\tau)^{-1 / 2} d \tau .
$$

Equation (24) can be written in the form;

$$
k C_{x=0}=\psi-2 \int_{0}^{\theta}(d \mu / d \rho)(\theta-\rho)^{-1 / 2} d \rho .
$$

On the assumption of the series equation of eq. (13), eq. (26) is obtained from eq. (25).

$$
k C_{x=0}=\psi-\theta^{-1 / 2} \sum_{j=1}^{\infty} j a_{j} \theta^{j / 2} B(j / 2,1 / 2) .
$$

Equation (26) can be written in the form;

$$
\begin{aligned}
C_{x=0} / C_{0}= & -(1 / \psi) \sum_{j=1}^{\infty} j a_{j} \eta^{(j-1) / 2} t^{(j-1) / 2} \\
& \times B(j / 2,1 / 2) .
\end{aligned}
$$

The change in $C_{x=0}$ with annealing time is calculated using the values of $a_{j}$ obtained from eqs. (18), (19), (20) and (21). As shown in Fig. 7, the change in $C_{x=0}$ is small and $C_{x=0}$ is always very close to $C_{0}$. This means that segregant is supplied to the surface by the diffusion with a sufficient velocity.

\section{Effect of the bulk concentration}

Equation (23) shows that the segregation

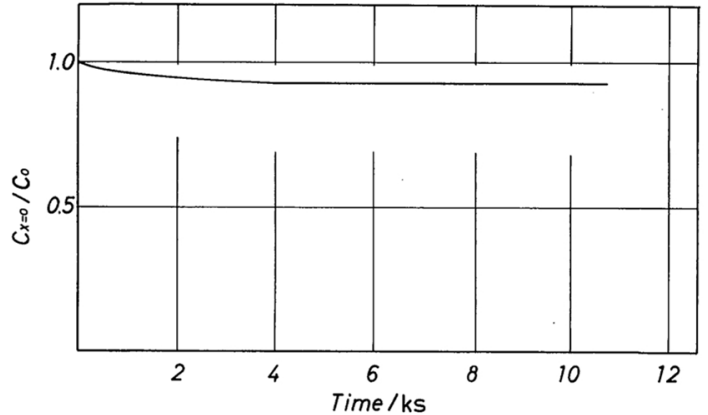

Fig. 7 The time dependence of $C_{x=0}$.

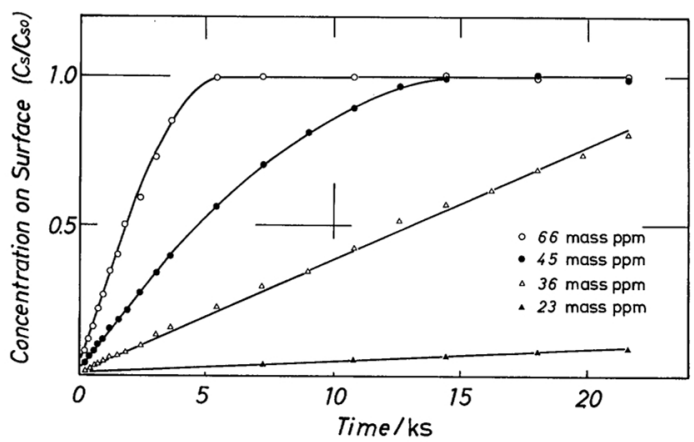

Fig. 8 The segregation behavior of sulfur in various bulk concentrations.

rate at the initial stage is in proportion to the bulk concentration. Figure 8 shows the relation between the segregation behavior and the bulk concentration of sulfur. From this figure, it is seen that the segregation rate becomes greater as the bulk concentration increases. The segregation rates at the initial stage are plotted against the bulk concentration. The result is shown as the solid line in Fig. 9. Actually, however, a movable sulfur concentration is smaller than the nominal bulk concentration, because sulfur is trapped by manganese or magnesium atoms which are impurities in the iron single crystal ${ }^{(3)}$. Therefore, the trapping effect by manganese is considered as a typical example;

$$
\mathrm{Mn}+\mathrm{S} \rightleftarrows \mathrm{MnS} .
$$

Therefore,

$$
a_{\mathrm{MnS}} / a_{\mathrm{Mn}(\mathrm{f})}=\exp \left(-\Delta G^{0} / R T\right),
$$

where $a_{\mathrm{MnS}}$ is the activity of $\mathrm{MnS}$, and $a_{\mathrm{Mn}(\mathrm{f})}$ and $a_{\mathrm{S}(\mathrm{f})}$ are the activities of movable manganese and sulfur, respectively, and $\Delta G^{0}$ is 


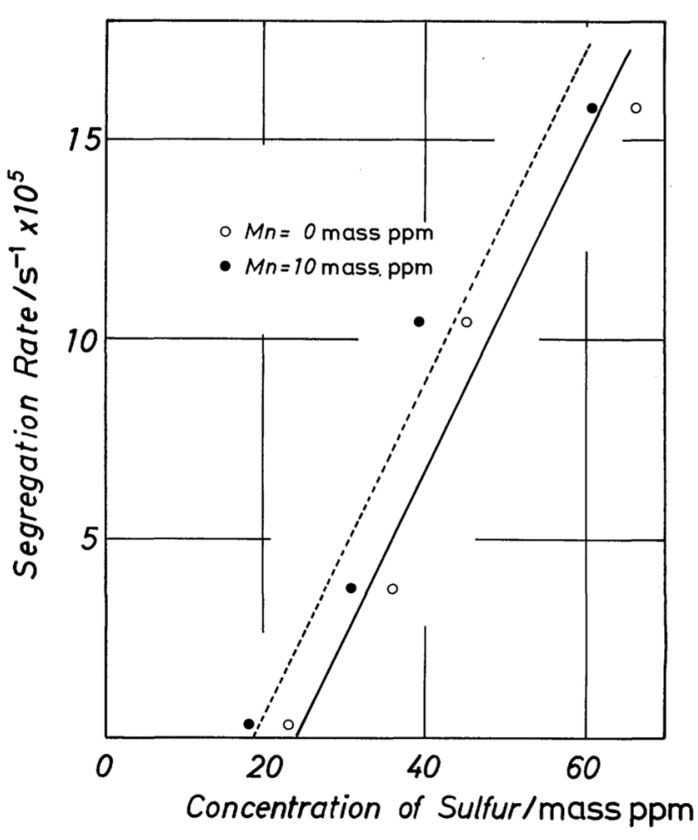

Fig. 9 The bulk concentration dependence of the segregation rate.

the standard free energy of formation of $\mathrm{MnS}$ which was published by Kubaschewski et al. ${ }^{(6)}$. Because MnS precipitates in the bulk,

$$
a_{\mathrm{MnS}}=1 \text {. }
$$

$a_{\mathrm{Mn}(\mathrm{f})}$ and $a_{\mathrm{S}(\mathrm{f})}$ are expressed approximately as follows:

$$
a_{\mathrm{Mn}(\mathrm{f})} \simeq C_{\mathrm{Mn}(\mathrm{f})}
$$

and

$$
a_{\mathrm{S}(\mathrm{f})} \simeq C_{\mathrm{o}(\mathrm{f})},
$$

where $C_{\mathrm{Mn}(\mathrm{f})}$ and $C_{\mathrm{o}(\mathrm{f})}$ are the concentrations of movable manganese and sulfur, respectively. Substituting eqs. (30), (31) and (32) for eq. (29) gives

$$
C_{\mathrm{Mn}(\mathrm{f})} \cdot C_{\mathrm{o}(\mathrm{f})}=\exp \left(\Delta G^{0} / R T\right) .
$$

Then, the following relations are given;

$$
C_{\mathrm{o}(\mathrm{f})}=C_{\mathrm{o}}-C_{\mathrm{MnS}}
$$

and

$$
C_{\mathrm{Mn}(\mathrm{f})}=C_{\mathrm{Mn}}-C_{\mathrm{MnS}} \text {, }
$$

where $C_{\mathrm{MnS}}, C_{\mathrm{Mn}}$ and $C_{\mathrm{o}}$ are the bulk concentrations of $\mathrm{MnS}$, manganese and sulfur, respectively. Equations (33), (34) and (35) can be combined to give eq. (36) for $C_{\mathrm{o}(\mathrm{f})}$.

$$
\begin{aligned}
C_{\mathrm{o}(\mathrm{f})}= & \left(C_{\mathrm{o}}-C_{\mathrm{Mn}}\right) \\
& \times\left[1+\sqrt{1+\left\{4 \frac{\exp \left(\Delta G^{0} / R T\right)}{\left(C_{\mathrm{o}}-C_{\mathrm{Mn}}\right)^{2}}\right\}}\right] / 2 .
\end{aligned}
$$

From eq. (36), it is possible to calculate the concentration of movable sulfur, when the bulk concentration of manganese is assumed to be 10 mass ppm. The dotted line in Fig. 9 shows the relation between the segregation rate and the movable sulfur concentration. The dotted and solid lines are both linear. Therefore, it is shown that the segregation rate is proportional to the bulk concentration of segregant. It is impossible, however, to make clear the trapping effect from this calculation.

\section{Conclusion}

The segregation rate onto the (100) surface of iron single crystal, the bulk concentration of which was adjusted, was measured with AES and compared with the theoretical value.

(1) Assuming that the segregation rate is proportional to the number of vacant sites on the surface and the bulk concentration of segregant just beneath the surface, the following solution of Fick's second law is deduced.

$$
\begin{aligned}
C_{\mathrm{s}} / C_{\mathrm{so}}= & k C_{\mathrm{o}} t-\left(4 k^{2} C_{\mathrm{o}} C_{\mathrm{so}} / 3 \sqrt{\pi D}\right) t^{3 / 2} \\
& +\left\{k^{2} C_{\mathrm{o}}\left(k C_{\mathrm{so}}^{2}-D C_{\mathrm{o}}\right) / 2 D\right\} t^{2}-\cdots
\end{aligned}
$$

At the initial stage of segregation, this equation shows that the concentration of segregant on the surface increases in proportion to the annealing time, and that the segregation rate is proportional to the bulk concentration and independent of the volume diffusion coefficient of segregant.

(2) The following processes were necessary to observe the segregation behavior with good reproducibility: After the specimen has been well annealed at a measurement temperature, the surface should be sputtered, until the surface concentration of segregant becomes equal to the bulk concentration of segregant.

(3) At the initial stage of segregation, the concentration of sulfur on the surface was observed to increase in proportion to the annealing time and reached a constant value. The segregation rate was proportional to the 
bulk concentration. These results coincide well with those of theoretical calculation.

\section{Acknowledgments}

The authors are indebted to Dr. M. Kurahashi for the calculation of the thoeretical equation and also to Dr. T. Takeuchi for offering the iron sigle crystal.

\section{REFERENCES}

(1) D. McLean: Grain Boundary in Metals, Clarendon
Press, Oxford, (1957), p. 131.

(2) W. H. Reinmuth: J. Phys. Chem., 65 (1961), 473.

(3) K. Yoshihara, M. Kurahashi and K. Nii: J. Japan Inst. Metals, 43 (1979), 409 (in Japanese); Trans. Japan Inst. Metals, 21 (1980), 425.

(4) H. S. Carlslaw and J. C. Jaeger: Conduction of Heat in Solids, Oxford University Press, London, (1947), p. 57.

(5) N. G. Ainslie and A. U. Seybolt: J. Iron Steel Inst., 194 (1960), 341.

(6) O. Kubaschewski, E. Ll. Evans and C. B. Alcock: Metallurgical Thermochemistry, Pergamon Press, England, (1967), p. 425. 